\title{
Cluster Detection Schemes in Spatio Temporal NeTWORKS
}

\author{
Midde Ranjit Reddy ${ }^{1}$, K. G. Srinivasa ${ }^{2}$ and B. Eswara Reddy ${ }^{3}$ \\ ${ }^{1}$ Research Scholar, CSE, JNTUA, Andhra Pradesh, India \\ ${ }^{2}$ Associate Professor, IT, CBPGEC, New Delhi, India \\ ${ }^{3}$ Professor, CSE, JNTUA, Andhra Pradesh, India.
}

\begin{abstract}
A spatiotemporal challenge can be portrayed as an inquiry that has no short of what one spatial and one momentary property. The spatial properties are region and geometry of the inquiry. The transient property is timestamp or time interval for which the challenge is real. The spatio fleeting inquiry as a general rule contains spatial, common and topical or non-spatial properties. Instances of such inquiries are moving auto, forest fire, and earth shake. Spatiotemporal educational accumulations essentially find changing estimations of spatial and topical attributes over a time allotment. Spatio transient bunching is a procedure of collection articles in view of their spatial and worldly similitude. It is generally new sub-field of information mining which increased high notoriety particularly in geographic data sciences because of the inescapability of a wide range of area based or ecological gadgets that record position, time or/and natural properties of a protest or set of articles progressively. As a result, distinctive sorts and a lot of spatio-transient information got to be distinctly accessible that acquaint new difficulties with information examination and require novel ways to deal with learning revelation.
\end{abstract}

\section{KEYWORDS}

Cluster, DBSCAN, FCM, Partition, Hidden Markov Models

\section{INTRODUCTION}

A spatiotemporal protest can be characterized as a question that has no less than one spatial and one fleeting property. The spatial properties are area and geometry of the question. The transient property is timestamp or time interim for which the protest is legitimate. The spatio temporal question more often than not contains spatial, worldly and topical or non-spatial properties. Cases of such questions are moving auto, woodland fire, and earth shake. Spatiotemporal informational collections basically catch changing estimations of spatial and topical traits over a timeframe. An occasion in a spatiotemporal dataset portrays a spatial and worldly wonder that may occurs at a specific time $t$ and area $x$. Cases of occasion sorts are earth tremor, typhoons, street activity stick and street mischance. In certifiable a significant number of these occasions interface with each other and show spatial and transient examples which may comprehend the physical marvel behind them. Hence, it is essential to recognize effectively the spatial and fleeting components of these occasions and their connections from vast spatiotemporal datasets of a given application area. Spatiotemporal examination can be ordered as worldly information investigation, spatial information examination, dynamic spatiotemporal information investigation and static spatiotemporal information examination. The worldly information investigation settles the spatial measurement and breaks down how topical qualities information change with time. Investigation of precipitation, temperature and mugginess of a given locale over a timeframe is a case of this kind. The spatial information investigation breaks down how topical properties data changing as for a separation from a spatial reference at a predefined time. Investigation of progress in temperature and dampness values while moving far from ocean drift at a given time is a case of this sort. The element spatiotemporal information examination fixes topical traits measurement

DOI: $10.5121 / \mathrm{ijdkp} .2018 .8202$ 
and dissects how spatial properties change with time. Investigation of moving auto information, spread of flame are cases of this class. The static spatiotemporal information investigation settles the transient and topical property measurements and studies the spatial measurement. A case of this is discovering areas having same precipitation at same time. Investigation of substantial volume of spatiotemporal information without settling any measurement is extremely troublesome and complex. However the information mining can be utilized to reveal obscure examples and patterns inside the information.

\section{RELATED WORK}

Bunching is one of the real information mining strategies in expansive databases for learning disclosure. It is the way toward gathering substantial informational indexes as indicated by their closeness. Spatial information records data with respect to the area, shape and its impact on elements. At the point when such information is time variation, it is called spatio-fleeting information. Spatio-worldly information mining is an up and coming exploration point which concentrates on considering and actualizing novel computational systems for expansive scale spatio transient information [1]. Spatio-transient grouping calculations [2] need to consider the spatial and worldly neighbors of articles while separating the bunches. In genuine applications, we encounter different sorts of spatio-worldly information. [3] isolated spatio-transient information into five classes including spatio-fleeting occasions, geo referenced factors, geo referenced time arrangement, moving items, and directions. In spatio-transient occasion information, there is an arrangement of occasions, each happened in a spatial area and accompanying its timestamp. Bunching this kind of information intends to locate an arrangement of occasions that are near each other in both space and time. One of the generally utilized strategies for grouping these sorts of information is output measurements [4], [5]. In this technique, one moves a tube shaped window of variable size and shape, over a geological district to identify bunches of occasions with the most elevated probability proportions. In [6], a broadened adaptation of FCM has been proposed to discover round groups of hotspots in spatiofleeting topographical data framework information. For each timestamp, the occasions are grouped in view of their spatial area, and after that, an examination between happened bunches in back to back time stamps has been performed to close a few translations about occasions. As in [7] proposed two spatio-worldly grouping techniques, which are called ST-GRID and STDBSCAN, to identify seismic occasions in China and neighboring nations. The ST-GRID technique utilized a multidimensional framework that covers the whole spatio-fleeting component space. At that point, by combining the thick neighbor cells, spatio-worldly groups were framed. ST-DBSCAN amplified DBSCAN by rethinking thickness reach ability utilizing spatial and worldly span. Both strategies abused a requested k-dist diagram [8] to decide their parameters. Georeferenced time arrangement are made out of an arrangement of settled land facilitates, each comparing to at least one time arrangement. Georeferenced factors information frame a unique instance of georeferenced time arrangement where just the latest purpose of time arrangement is accessible. Bunching this kind of information expects to gathering objects in view of their spatial closeness and transient likenesses. In [9], FCM has been utilized to bunch climate time arrangement. The Pearson relationship coefficient was utilized as the likeness measure communicating closeness of two time arrangement and a technique to decide the quantity of groups has been proposed. In any case, the strategy does not include the spatial piece of information in the grouping procedure. As in [10] proposed a thickness based spatio-transient grouping. In this technique, a spatial proximate system has been developed utilizing Delaunay triangulation and a spatio-transient autocorrelation examination was utilized to characterize the spatio-fleeting neighborhood. In [11], a broadened adaptation of FCM was proposed for picture division by considering the spatial area of pixels. This strategy has been considered [12] for bunching spatio-worldly information. In this approach, a spatial punishment term that was 
ascertained utilizing a spatial contiguity network has been added to the target capacity to ensure an estimated spatial homogeneity of the groups. As in [13] proposed ST-DBSCAN adjustment of DBSCAN with idea of thickness component which is level of thickness of group. It finds groups as indicated by non-spatial, spatial and worldly estimations of the articles. Attributable to the expansion in movement among real urban communities, there was a squeezing need a proficient activity administration framework set up to determine such issues. Here, the expression "proficient" infers that the framework ought to fuse the most recent innovations and ought to likewise be practical strategy to deal with the steady surges of activity information that is coming into the framework. A review by Jim Forsyth found that a normal American spends around $42 \mathrm{~h}$ stuck in movement every year. Encourage, the time taken to drive has turned out to be more than twice of what it was couple of decades back in the 1980s. The measure of time squandered stuck in activity alone was around 6.6 billion hours in 2007 and the sum lost was around $160 \$$ billion in time and fuel while the review puts appraise for the time squandered in year 2020 at around 8.8 billion hours and the cash at $192 \$$ billion. That is a considerable measure of time and cash that goes to the deplete for being basically stuck in movement. There are a few thickness based bunching calculations, as in [14] proposed the Density Based grouping calculation for finding groups from database with clamor. The calculation uses to measures viz., MinPts and Epf. The MinPts goes about as the base limit an incentive for the quantity of focuses to be available inside the area given the Epf span.

As in [15] proposed FN-Descant calculation those utilizations Fuzzy changes to the first DBScan calculation to gauge activity blockage levels. The calculation was tried on information that was gotten from an auto test which was sent for information obtaining. The grouping results were that six diverse movement blockage levels were acquired for the given information. Considering the activity models with the expansion in measure of information and the extent of capacity limit of huge limit, the measure of information that is being utilized as a part of the information mining applications is quickly expanding, in this manner making a need for proficient frameworks that make most extreme use of this information. There have been many activity administration frameworks set up however none of them can deal with live gushing information which makes them possibly un-valuable for constant condition questioning [16]. As in [17] analyzes different calculations, for example, DBScan, KMeans, Auto class for grouping system information where he talks about the advantages of one calculation over another and it presumes that the Auto class calculation has the best general exactness while k-Means calculation has a considerably quicker model building time regardless of having a marginally less precision than Auto class calculation however the DBScan calculation has a more noteworthy potential since it has the greater part of the associations in a littler subset of the bunches. In an Incremental Approach, bunching calculation is being actualized to group the clumps of online information that is coming through. A few people have already chipped away at incremental usage of grouping calculation. As in [14] concocted a technique to execute bunching calculation on a dataset incrementally. However as in [15] depict another incremental DBScan calculation that utilizations typical execution of the calculation for the main bunch and later the new group is converged with the old bunch as shown in Figure 1. The benefit of this calculation is that it can add indicates in mass that groups that were framed at first. There have been distinctive ways to deal with handle bunching on ongoing on the web/stream information. As in [1] proposed Clustered-a grouping calculation for developing stream information. The calculation has the K-Means approach at its center and its system includes utilizing on the web segment and disconnected part. The online segment stores the rundown of the measurable investigation of the bunching comes about and the disconnected segment utilizes these factual examinations of the grouping comes about and the disconnected segment utilizes these measurable information to determine helpful data which is generally done by an expert. Numerous cross breed calculations are additionally accessible however its disadvantage will be its computational intricacy as it inserts more than one strategy, and that is its preference [3]. As in [10] proposed D-Stream-a calculation for grouping stream information 
utilizing a thickness based approach. The calculation has two unique segments viz. an on the web and a disconnected segment. The online segment is utilized to delineate info information onto a lattice based structure and the disconnected segment then computes the densities of the frameworks and performs bunching on the information based the densities of the matrices.

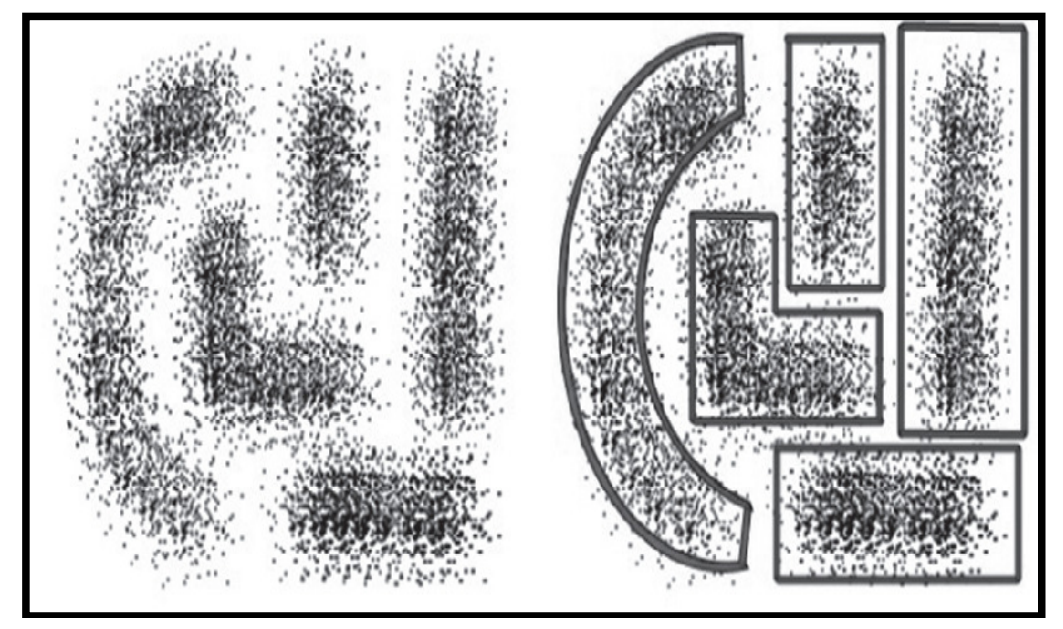

Figure 1. DBSCAN

Unsupervised order, or bunching, gets structure from information by utilizing target criteria to parcel the information into homogeneous gatherings so that the inside gathering object similitude and the between gathering object disparity are upgraded all the while [8]. Classification and translation of structure are accomplished by investigating the models developed regarding the element esteem dispersions inside each gathering. Previously, group examination strategies have concentrated on information portrayed by static components, i.e., estimation of the element does not change, or the change is insignificant, after some time. In numerous true applications, that cover various areas, for example, building frameworks, human physiology, and monetary and social frameworks, the dynamic attributes, i.e., how a framework interfaces with nature and advances after some time, are of intrigue. Dynamic normal for these frameworks is best depicted by worldly elements whose qualities change fundamentally amid the perception time frame. Bunching transient information is intrinsically more unpredictable than grouping static information on the grounds that:

(i) the dimensionality of information is essentially bigger, and (ii)the demonstrate structures that depict singular bunch structures and more mind boggling and harder to break down and translate.

The creator proposed, expect the fleeting information arrangements that characterize the dynamic qualities of the wonder under review fulfill the Markov property, and the information era might be seen as a probabilistic stroll through a settled arrangement of states. At the point when state definitions straightforwardly compare to highlight values, a Markov chain show representation of the information might be fitting [9]. At the point when the state definitions are not specifically detectable, or it is not possible to characterize states by thorough list of highlight qualities (e.g., when the information is portrayed with various constant esteemed worldly components), a Hidden Markov show (HMM) representation is more suitable. In this paper, the HMM strategy is connected for unsupervised learning of fleeting information. Consider the case of ceaseless discourse acknowledgment. Strategies have been produced [10] for dissecting the discourse range and removing worldly components from a consistent discourse flag. Gee characterize include groupings that compare to sub word units. Lexical and semantic principles are then utilized to perceive full sentences. A considerable measure of foundation learning as phonemes and syllable 
for disconnected word acknowledgment, telephone like units (PLUs) for sub-word acknowledgment and syllable and acoustic units for sentence level acknowledgment have been produced through years of thorough examination. In this way, the HMM structures utilized for acknowledgment are very much characterized, and the discourse acknowledgment errand is lessened to learning model parameters for proficient and strong acknowledgment. Then again, when one considers issues, for example, helpful impacts of another therapeutic strategy or examination of securities exchange information, very little is thought about models that can be utilized to structure, break down, and translate accessible information. To address these issues, need to create approaches that empower us to get alert models from transient information. We will probably create through the HMM models got from accessible information, a precise and reasonable representation of framework flow in a given space. It is imperative for our grouping framework to decide the best segment, i.e., number of bunches in the information, and best model structure, i.e., the quantity of states in a model. The initial step permits us to separate the information into homogeneous gatherings, and the second step gives a precise state based representation of the dynamic marvels comparing to each gathering. The assignments of this approach are by [11]. The target of Descriptive and generative model-based bunching is to infer a worldwide model fit for portraying the entire dataset. Some of these strategies depend on a meaning of multivariate thickness circulation and search for an arrangement of fitting parameters for the model. In Gaffney and Smyth (1999) it is proposed a grouping technique in light of a blend display for ceaseless directions. The directions are spoken to as practical information, i.e. every individual is displayed as an arrangement of estimation given by a component of time contingent upon an arrangement of parameters that models the collaboration of the distinctive disseminations. The articles that are probably going to be produced from a center direction in addition to Gaussian commotion are assembled together by method for the EM calculation. In a progressive work, spatial and worldly move of directions inside each group is likewise considered. Another approach in light of a model-based strategy is introduced, where the delegate of a bunch is communicated by method for a Markov model that gauges the move between progressive positions. The parameter estimation assignment for the model is performed by method for EM calculation. Another way to deal with bunch complex type of information, similar to directions, is to change the mind boggling objects into elements vectors, i.e. an arrangement of multidimensional vectors where each measurement speaks to a solitary normal for the first protest, and after that to bunch them utilizing non specific grouping calculations, as, for instance, k-implies as shown in Figure 2.

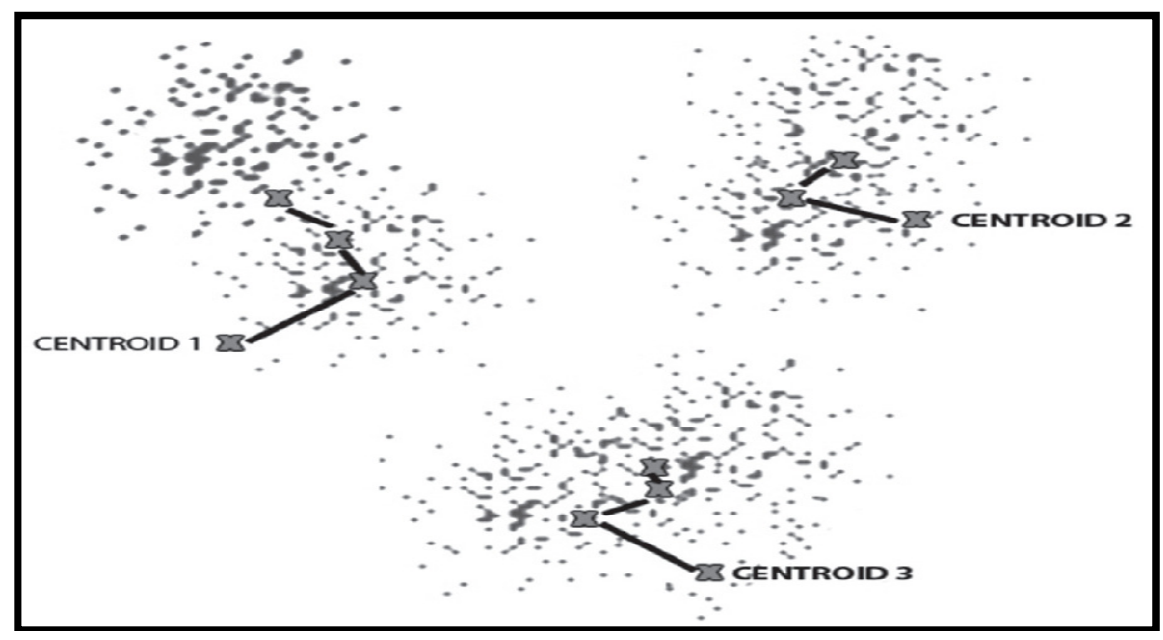

Figure 2. K-means 
Be that as it may, the mind boggling structure of the directions not always permits an approach of this kind, since the greater part of these strategies require that every one of the vectors be of a similar length. In actuality, one of a great extent received way to deal with the grouping of directions comprises in characterizing separation works that epitomize the idea of similitude among the information things. The thickness based grouping strategies utilize a thickness limit around each protest recognize the intriguing information things from the clamor. DBSCAN one of the principal case of thickness based bunching, visits the entire dataset and labels each protest either as center question (i.e. a question that is conclusively inside a group), fringe protest (i.e. objects at the fringe of a group), or clamor (i.e. objects completely outside any group). The approach of picking a bunching technique and a separation capacity is only a beginning stage for a more evolutes way to deal with mining. For instance, in the fundamental idea of the separation capacity is misused to push the significance of the worldly attributes of directions. The creators propose another approach called transient centering to better adventure the fleeting perspective and enhance the nature of direction bunching. For instance, two directions might be altogether different if the entire time interim is considered. In any case, if just a little sub-interim is viewed as, these directions might be discovered fundamentally the same as. Subsequently, it is exceptionally essential for the calculation to productively deal with various spatial and worldly granularities. As said by the creators, normally a few sections of directions are more imperative than others. It is proposed an alternate approach, where the directions are spoken to as piece-wise portions, potentially with missing interims. The proposed technique attempt to decide a nearby time interim, i.e. a maximal time interim where every one of the directions are match shrewd near each other. The likeness of directions depends on the measure of time in which directions are close and the mining issue is to discover all the direction assembles that is close inside a given edge. The directions are spoken to as groupings of focuses without unequivocal worldly data and they are parcelled into an arrangement of semi straight fragments. Every one of the sections is gathered by method for a thickness based bunching technique and an agent direction for each group is resolved. Introducing a calculation to discover visit development designs that speak to aggregate conduct of moving articles where an example, called T-example, was characterized as a grouping of focuses with fleeting moves between continuous focuses. A T-example is found if its spatial and fleeting parts roughly compare to the information arrangements (directions). The importance of these examples is that distinctive items visit similar spots with comparable time interims. Once the examples are found, the established succession mining calculations can be connected to discover visit designs. Critical to the assurance of T-examples is the meaning of the meeting locales. For this, the Region-of-Interest (RoI) thought was proposed. A RoI is characterized as a place went by many items. Moreover, the length of stay can be considered. The thought behind Roi is to partition the working area into cells and check the quantity of directions.

\section{CONCLUSIONS}

Spatiotemporal examination can be requested as common data examination, spatial data examination, dynamic spatiotemporal data examination and static spatiotemporal data examination. The common data examination settles the spatial estimation and separates how topical characteristics data change with time. Examination of precipitation, temperature and moisture of a given area over a time period is an instance of this kind. This paper examines distinctive methodologies for bunching of spatial time objects. The primary perception we have done here is the manner by which to distinguish the bunches. There are diverse methods talked about before. Each procedure has focal points and disservices. This paper is valuable to the scientists who are intrigued to take a shot at bunch location issues. 
International Journal of Data Mining \& Knowledge Management Process (IJDKP) Vol.8, No.2, March 2018

\section{REFERENCES}

[1]. John F. Roddick and Myra Spiliopoulou. A survey of temporal knowledge discovery paradigms and methods. IEEE Trans. on Knowl. and Data Eng., 14(4):750-767, ISSN 1041- 4347, July 2002.

[2]. Manso, J. A. ; Times, V. C. ; Oliveira, G. ; Alvares, Luis Otavio ; Bogorny, V. "DB-SMoT: A Direction-Based Spatio-Temporal Clustering Method" In: IEEE International Conference on Intelligent Systems (IS), 2010, Londres. Proceedings of the IEEE International Conference on Intelligent Systems.

[3]. S. Kisilevich, F. Mansmann, M. Nanni, and S. Rinzivillo, "Spatiotemporal clustering," in Data mining and Knowledge Discovery Handbook. New York: Springer, 2010, pp. 855-874

[4]. M. Kulldorff, "Prospective time periodic geographical disease surveillance using a scan statistic," J. Roy. Statist. Soc. A, vol. 164, no. 1, pp. 61-72, 2001.

[5]. H. Izakian and W. Pedrycz, "A new PSO-optimized geometry of spatial and spatio-temporal scan statistics for disease outbreak detection," Swarm Evol. Comput., vol. 4, pp. 1-11, Jun. 2012.

[6]. F. Di Martino and S. Sessa, "The extended fuzzy C-means algorithm for hotspots in spatio-temporal GIS,” Expert Syst. Appl., vol. 38, no. 9, pp. 11829-11836, Sep. 2011.

[7]. M. Wang, A. Wang, and A. Li, "Mining spatial-temporal clusters from geo-databases," in Proc. 2nd Int. Conf. Adv. Data Mining Appl., 2006, pp. 63-270.

[8]. M. Ester, H. P. Kriegel, J. Sander, and X. Xu, "A density-based algorithm for discovering clusters in large spatial databases with noise,” Data Mining Knowl. Discovery, pp. 226-231, 1996.

[9]. Z. Liu and R. George, "Fuzzy cluster analysis of spatio-temporal data," in Proc. 18th Int. Symp. Comput. Inf. Sci., Antalya, Turkey, 2003, pp. 984- 991.

[10]. M. Deng, Q. Liu, J. Wang, and Y. Shi, “A general method of spatiotemporal clustering analysis," Sci. Chin. Inf. Sci., pp. 1-14, 2011.

[11]. R. Coppi, P. D’Urso, and P. Giordani, "A fuzzy clustering model for multivariate spatial time series," J. Classif., vol. 27, no. 1, pp. 54-88, Mar. 2010.

[12]. Derya Birant \& Alp Kut, (2007) "ST-DBSCAN: An algorithm for clustering spatio-temporal data", Data \& Knowledge Engineering, Volume 60, Issue 1, January, Pages 208-221.

[13]. Chan KP, chee Fu AW (1999) Efficient time series matching by wavelets. In: In ICDE, pp 126-133

[14]. Ester, M., Kriegel, H.P., Sander, J., Xu, X.: A density-based algorithm for discovering clusters in large spatial databases with noise. Kdd 96(34), 226-231 (1996)

[15]. Diker, A.C., Nasibov, E.: Estimation of traffic congestion level via fn-dbscan algorithm by using GPS data. In: Problems of Cybernetics and Informatics (PCI), 2012 IV International Conference, pp. 1-4. IEEE (2012)

[16]. Bertini, R.L., El-Geneidy, A.: Advanced traffic management system data. In: Assessing the Benefits and Costs of ITS, pp. 287-314. Springer, Berlin (2004)

[17] .Erman, J., Arlitt, M., Mahanti, A.: Traffic classification using clustering algorithms. In: Proceedings of the 2006 SIGCOMM Workshop on Mining Network Data, pp. 281-286. ACM (2006) 